\title{
Determining a quantum state by means of a single apparatus.
}

\author{
A.E. Allahverdyan ${ }^{1,2)}$, R. Balian ${ }^{3)}$ and Th.M. Nieuwenhuizen ${ }^{1)}$ \\ 1) Institute for Theoretical Physics, Valckenierstraat 65, 1018 XE Amsterdam, The Netherlands \\ ${ }^{2)}$ Yerevan Physics Institute, Alikhanian Brothers St. 2, Yerevan 375036, Armenia and \\ 3) SPhT, CEA-Saclay, 91191 Gif-sur-Yvette cedex, France
}

\begin{abstract}
The unknown state $\hat{\rho}$ of a quantum system $\mathrm{S}$ is determined by letting it interact with an auxiliary system A, the initial state of which is known. A one-to-one mapping can thus be realized between the density matrix $\hat{\rho}$ and the probabilities of occurrence of the eigenvalues of a single and factorized observable of $\mathrm{S}+\mathrm{A}$, so that $\hat{\rho}$ can be determined by repeated measurements using a single apparatus. If $\mathrm{S}$ and $\mathrm{A}$ are spins, it suffices to measure simultaneously their $z$-components after a controlled interaction. The most robust setups are determined in this case, for an initially pure or a completely disordered state of A. They involve an Ising or anisotropic Heisenberg coupling and an external field.
\end{abstract}

Consider a set of identical quantum systems $\mathrm{S}$, prepared in some unknown state $\hat{\rho}$. Information on $\hat{\rho}$ can be gathered by measuring some observable $\hat{\omega}$ of S. Such a measurement has a statistical nature, whether $\hat{\rho}$ is pure or not: when repeated many times, it provides the probabilities $p_{i}=\operatorname{tr} \hat{\rho} \hat{\pi}_{i}$ for the distinct eigenvalues $\omega_{i}$ of an operator $\hat{\omega}$, where $\hat{\pi}_{i}$ are the associated eigenprojections. We thus find partial information on $\hat{\rho}$. A question then arises which lies at the heart of quantum theory: Which observables need to be measured for a complete determination of $\hat{\rho}$ 1,2.3.4 ? The recognition that certain non-commuting observables have to be measured for that purpose was used by Bohr to formulate the principle of complementarity ${ }^{1.5}$. Later on the problem of determining an unknown state was considered from various perspectives for continuous ${ }^{3}$ and discrete systems ${ }^{4}$ and found applications in quantum communication ${ }^{6.7}$. However, during all these developments it was not questioned whether non-commutative measurements are truly needed.

Here we face the problem of determining from some collection of experimental data the whole set of unknown matrix elements of the state $\hat{\rho}$ of a system S. In fact, what we call "the state of a system" refers to a density matrix describing an ensemble, and the determination of the data will require repeated experiments. However, we will show that it is sufficient for our purposes to produce these data by means of a single apparatus which measures commuting observables only. The key of the method consists in coupling the system $\mathrm{S}$ to an auxiliary system $A$, the state of which we know. We will show that the full $\hat{\rho}$ can then be deduced from simultaneous measurements of two obviously commuting observables, $\hat{\omega}$ pertaining to $\mathrm{S}$ and $\hat{o}$ pertaining to $\mathrm{A}$, respectively. Repeated measurements of $\hat{\omega}$ and $\hat{o}$ yield the joint probabilities of occurrence for all pairs of eigenvalues of $\hat{\omega}$ and $\hat{o}$; this will be sufficient to determine the whole density matrix $\hat{\rho}$ of $\mathrm{S}$, provided the initial state of $\mathrm{A}$ and the joint evolution of $\mathrm{S}+\mathrm{A}$ are known. We shall discuss the most robust measurements of this type and point out certain advantages that this scheme provides over the usual methods of state determination. Realistic experiments can be designed along these lines, as we shall see.

General reasoning. The following counting argument already suggests the feasibility of the above idea: The number of real parameters to be determined for finding $\hat{\rho}$ which lives in $m$-dimensional Hilbert space is $m^{2}-1$, since $\hat{\rho}$ is a $m \times m$ hermitian matrix with unit trace. On the other hand, the most informative repeated measurements of an observable $\hat{\omega}$ of $\mathrm{S}$ are those for which the spectrum $\omega_{i}$ is non-degenerate; they provide $m-1$ independent data, the probabilities $\operatorname{tr} \hat{\rho} \hat{\pi}_{i}, i \leq 1 \leq m$. Thus, if measurements are performed on $\mathrm{S}$ only, they must deal with at least $\left(m^{2}-1\right) /(m-1)=m+1$ non-commuting observables in order to fully determine the unknown $\hat{\rho}$. For instance, the $2 \times 2$ density matrix $\hat{\rho}=\frac{1}{2}(\hat{1}+\vec{\rho} \cdot \overrightarrow{\hat{\sigma}})$ of a spin- $\frac{1}{2}$ is parameterized by the $m^{2}-1=3$ expectation values $\vec{\rho}=\operatorname{tr}(\hat{\rho} \cdot \overrightarrow{\hat{\sigma}})$ of the Pauli matrices $\overrightarrow{\hat{\sigma}}=\left(\hat{\sigma}_{1}, \hat{\sigma}_{2}, \hat{\sigma}_{3}\right)$ with $\hat{\sigma}_{1} \hat{\sigma}_{2}=i \hat{\sigma}_{3}$, and its determination requires measuring the spin in $m+1=3$ non-coplanar directions. Experiments with three different apparatuses are thus needed. This conclusion holds even if we are given the information that the unknown state $\hat{\rho}$ is pure $\left(\hat{\rho}^{2}=\hat{\rho}\right.$ or $\left.\vec{\rho}^{2}=1\right)$, since then the measurement of two components of $\vec{\rho}$ does not fix the sign of the third one. For $m \geq 3$ the construction of the needed $m+1$ non-commuting observables is less straightforward, see the discussion in $\operatorname{Ref}^{4}$.

In order to design a scheme where $\hat{\rho}$ will be determined by measurements of a single observable (or, equivalently, by commuting measurements only), it is natural to introduce $\stackrel{8}{-}$ an auxiliary system A that we term the assistant, and which lies in a known state $\hat{r}$. Let $n$ be the number of dimensions of the Hilbert space of A. The compound system $\mathrm{S}+\mathrm{A}$ has a $m n \times m n$ density matrix $\hat{\mathcal{R}}=\hat{\rho} \otimes \hat{r}$. Ref. ${ }^{8}$ proposes measuring one of its observables, a "universal quantum observable", $\hat{\Omega}=\sum \Omega_{\alpha} \hat{P}_{\alpha}$, where the spectrum $\Omega_{\alpha}$ is not degenerate so that the eigenprojections $\hat{P}_{\alpha}$ (with $1 \leq \alpha \leq m n$, noting that $\sum_{\alpha} \hat{P}_{\alpha}=\hat{1}$ ) constitute a complete set of $m n-1$ commuting observables. Such repeated measurements provide $m n-1$ independent data $P_{\alpha}=\operatorname{tr} \hat{\mathcal{R}} \hat{P}_{\alpha}$, the probabilities of the eigenvalues $\Omega_{\alpha}$ of $\hat{\Omega}$. The set $P_{\alpha}$ also represents the diagonal elements of the density matrix $\hat{\rho} \otimes \hat{r}$ in the basis that diagonalizes $\hat{\Omega}$. A linear mapping $\hat{\rho} \mapsto P_{\alpha}=\sum_{i j} R_{\alpha, i j} \rho_{j i}$ is thus generated, leading from the $m^{2}-1$ real parameters of $\hat{\rho}$ to the $m n-1$ data $P_{\alpha}$. If the assistant $\mathrm{A}$ has the same dimensionality $m$ as $\mathrm{S}$, this mapping is repre- 
sented by a square matrix. In general, if the measured observable $\hat{\Omega}$ intertwines sufficiently $\mathrm{S}$ and $\mathrm{A}$, the determinant $\Delta=\operatorname{det} R_{\alpha, i j}$ of this matrix may be expected to be non-zero. The inverse mapping then solves our problem: measurements of $\hat{\Omega}$, performed repeatedly with a single apparatus, yield the probabilities $P_{\alpha}$, the knowledge of which is equivalent to that of $\hat{\rho}$. If $n>m$, the set $P_{\alpha}$ can still determine $\hat{\rho}$, but it is overabundant.

However, the above scheme is not easy to implement in practice, since it implies measuring an observable $\hat{\Omega}$ (or a commuting set $\hat{P}_{\alpha}$ ) which thoroughly mixes $\mathrm{S}$ and A. We propose here a modified procedure, which will allow a much simpler choice for $\hat{\Omega}$. The measurement of $\hat{\Omega}$ is performed not at the time $t=0$, at which S is prepared in the unknown state $\hat{\rho}$ and $\mathrm{A}$ in the known state $\hat{r}$, but at a later time $t=\tau$. During the lapse $0<t<\tau$, $\mathrm{S}$ and $\mathrm{A}$ interact, their evolution being generated by a known Hamiltonian $\hat{H}$. The state of the composite system $\mathrm{S}+\mathrm{A}$ which is tested is now $\hat{\mathcal{R}}_{\tau}=\hat{U} \hat{\mathcal{R}}_{0} \hat{U}^{\dagger}$, where the initial state is $\hat{\mathcal{R}}_{0}=\hat{\rho} \otimes \hat{r}$ and the evolution operator is $\hat{U}=\mathrm{e}^{-\mathrm{i} \hat{H} \tau}$. The required mixing of $\hat{\rho}$ and $\hat{r}$ being thus achieved by dynamics, we can now measure the simplest possible non-degenerate observable $\hat{\Omega}$, a factorized quantity $\hat{\Omega}=\hat{\omega} \otimes \hat{o}$. The observables $\hat{\omega}$ and $\hat{o}$ of $\mathrm{S}$ and A have the spectral decompositions $\hat{\omega}=\sum_{i=1}^{m} \omega_{i} \hat{\pi}_{i}$ and $\hat{o}=\sum_{a=1}^{n} o_{a} \hat{p}_{a}$ and the projection operator $\hat{P}_{\alpha}$, with $\alpha=\{i a\}$, takes the form $\hat{P}_{\alpha} \equiv \hat{P}_{i a}=\hat{\pi}_{i} \otimes \hat{p}_{a}$. Repeated measurements of $\hat{\Omega}$, that is, of $\hat{\omega}$ and $\hat{o}$ simultaneously, determine the joint probabilities

$$
P_{\alpha} \equiv P_{i a}=\operatorname{tr} \hat{U}(\hat{\rho} \otimes \hat{r}) \hat{U}^{\dagger}\left(\hat{\pi}_{i} \otimes \hat{p}_{a}\right)
$$

to observe $\omega_{i}$ for $\mathrm{S}$ and $o_{a}$ for $\mathrm{A}$. (The numbers $P_{i a}$ are the diagonal elements of $\hat{U}(\hat{\rho} \otimes \hat{r}) \hat{U}^{\dagger}$ in the factorized basis which diagonalizes $\hat{\omega}$ and $\hat{o}$.) Like above, the mapping $\hat{\rho} \mapsto P_{\alpha}$ is expected to be invertible for $n \geq m$, provided $\hat{H}$ couples $\mathrm{S}$ and A sufficiently. Then simultaneous measurements of $\hat{\omega}$ on the system $\mathrm{S}$ and of $\hat{o}$ on the assistant $\mathrm{A}$, based on the counting of the events $\{i a\}$ and of their correlations, fully determine $\hat{\rho}$ through inversion of the equation (11).

For given observables $\hat{\omega}$ and $\hat{o}$ and for a given initial state $\hat{r}$ of the assistant, the precision of this scheme of measurement of $\hat{\rho}$ relies on the ratio between the experimental uncertainty about the set $P_{\alpha}$ and the resulting uncertainty on $\hat{\rho}$, which can be characterized by the determinant $\Delta$ of the transformation (11). For $\Delta=0$ it would be impossible to determine $\hat{\rho}$ by means of $P_{\alpha}$. The Hamiltonian $\hat{H}$ and the duration $\tau$ of the interaction should thus be chosen so as to maximize $|\Delta|$.

Two by two density matrix. In the following we illustrate the above ideas by studying a two-level system $\mathrm{S}$ $(m=2)$. We exhibit in particular the best measurement schemes that correspond to the largest $|\Delta|$. We use the spin- $\frac{1}{2}$ representation $\hat{\rho}=\frac{1}{2}(\hat{1}+\vec{\rho} \cdot \overrightarrow{\hat{\sigma}})$. The determination of the unknown polarization vector $\vec{\rho}$ relies on the coupling of $\mathrm{S}$ with the assistant $\mathrm{A}$, which we first take as another two-level system $(n=2)$. The observables $\hat{\omega}$ and $\hat{o}$ to be measured are the $z$-components $\hat{\sigma}_{3}$ and $\hat{s}_{3}$ of $\mathrm{S}$ and $\mathrm{A}$, which may be equal to 1 or -1 . The projection operators are $\hat{\pi}_{i}=\frac{1}{2}\left(\hat{1} \pm \hat{\sigma}_{3}\right)$ and $\hat{p}_{a}=\frac{1}{2}\left(\hat{1} \pm \hat{s}_{3}\right)$ for $i$ and $a$ equal to \pm 1 . Experiments determine the four joint probabilities $P_{\alpha}=\left\{P_{++}, P_{+-}, P_{-+}, P_{--},\right\}$for $\sigma_{3}$ and $s_{3}$ to equal 1 or -1 . These probabilities are related to the three real parameters $\vec{\rho}$ of $\hat{\rho}$ through Eq. (1), which reads

$$
\begin{gathered}
P_{\alpha}=u_{\alpha}+\vec{v}_{\alpha} \cdot \vec{\rho}, \\
u_{\alpha}=\frac{1}{2}\left[\hat{U}(\hat{1} \otimes \hat{r}) \hat{U}^{\dagger}\right]_{\alpha, \alpha}, \quad \vec{v}_{\alpha}=\frac{1}{2}\left[\hat{U}(\hat{\vec{\sigma}} \otimes \hat{r}) \hat{U}^{\dagger}\right]_{\alpha, \alpha}
\end{gathered}
$$

with $\alpha=\{i a\}=\{ \pm \pm\}$ and matrix elements taken in the standard representation of the Pauli matrices $\hat{\vec{\sigma}}$ and $\hat{\vec{s}}$.

By construction, the mapping (2] 3) is such the the probabilities $P_{\alpha}$ are non-negative and normalized for any $\hat{\rho}$ such that $\vec{\rho}^{2} \leq 1$. These properties are expressed by

$$
u_{\alpha} \geq\left|\vec{v}_{\alpha}\right|, \quad \sum_{\alpha} u_{\alpha}=1, \quad \sum_{\alpha} \vec{v}_{\alpha}=0 .
$$

The determinant $\Delta$ of the transformation $\hat{\rho} \mapsto P_{\alpha}$ is four times the volume of the parallelepiped having any three of the four vectors $\vec{v}_{\alpha}$ as its sides, e.g., $\Delta=$ $4 \vec{v}_{++} \cdot\left(\vec{v}_{-+} \times \vec{v}_{+-}\right)$. Provided the evolution operator $\hat{U}$ is such that the vectors $\vec{v}_{\alpha}$ are not coplanar, the transformation (2) can be inverted, and $\hat{\rho}$ is deduced from the set $P_{\alpha}$ of classical probabilities. Alternatively, $\hat{\rho}$ is deduced from the knowledge of the expectation values $\left\langle\hat{\sigma}_{3}\right\rangle,\left\langle\hat{s}_{3}\right\rangle$ and $\left\langle\hat{\sigma}_{3} \hat{s}_{3}\right\rangle$ at the time $t=\tau$, which are simultaneously measurable and are in one-to-one correspondence with the set $P_{\alpha}$.

We first look for the upper bound of $|\Delta|$ implied by the conditions (4). First we note that $|\Delta|$ increases with $\left|\vec{v}_{\alpha}\right|$ for each $\alpha$. We therefore maximize $\Delta^{2}$ under the constraints $\sum_{\alpha}\left|\vec{v}_{\alpha}\right|=1$ and $\sum_{\alpha} \vec{v}_{\alpha}=0$, that we account for by means of Lagrange multipliers $\lambda$ and $\vec{\mu}$. Varying $\frac{1}{8} \Delta^{2}+\lambda \sum_{\alpha}\left|\vec{v}_{\alpha}\right|+\vec{\mu} \sum_{\alpha} \vec{v}_{\alpha}$ we find

$$
\Delta\left(\vec{v}_{+-} \times \vec{v}_{-+}\right)=\lambda\left(\frac{\vec{v}_{++}}{\left|\vec{v}_{++}\right|}-\frac{\vec{v}_{--}}{\left|\vec{v}_{--}\right|}\right)
$$

and other equations resulting from all permutations of the $\alpha$ 's. This yields symmetric solutions for which the four vectors $\vec{v}_{\alpha}, \alpha=\{ \pm \pm\}$ form a regular tetrahedron:

$$
u_{\alpha}=\left|\vec{v}_{\alpha}\right|=\frac{1}{4}, \quad \frac{\vec{v}_{\alpha} \cdot \vec{v}_{\beta}}{\left|\vec{v}_{\alpha}\right|\left|\vec{v}_{\beta}\right|}=-\frac{1}{3}, \quad \alpha \neq \beta .
$$

As could have been anticipated, the solutions are not unique: they follow one from another by rotation in the space of the spins $\vec{\sigma}$ and permutations of the indices $\alpha$. The corresponding upper bound for the modulus of the determinant is $|\Delta|=1 /(12 \sqrt{3})$.

Let us show that this upper bound can be reached, provided the initial state of the assistant is pure. We have to construct a unitary operator $\hat{U}$ that satisfies Eqs. [3] [6), and to find a Hamiltonian $\hat{H}$ and an interaction time $\tau$ such that $\hat{U}=e^{-i \hat{H} \tau}$. Let us exhibit an example of 
such a solution. We assume that the assistant is initially polarized in the $z$-direction, $\hat{r}=\hat{p}_{+}=\frac{1}{2}\left(1+\hat{\sigma}_{3}\right)$, and we orient the tetrahedron $\vec{v}_{\alpha}$ in the direction $\vec{v}_{+ \pm}=$ $( \pm 1,1, \pm 1) / 4 \sqrt{3}, \vec{v}_{- \pm}=( \pm 1,-1, \mp 1) / 4 \sqrt{3}$. The correspondence (2) then takes an especially simple form:

$$
\rho_{1}=\sqrt{3}\left\langle\hat{s}_{3}\right\rangle, \quad \rho_{2}=\sqrt{3}\left\langle\hat{\sigma}_{3}\right\rangle, \quad \rho_{3}=\sqrt{3}\left\langle\hat{\sigma}_{3} \hat{s}_{3}\right\rangle,
$$

yielding directly the density matrix $\hat{\rho}$ in terms of the expectation values and the correlation of the commuting observables $\hat{\sigma}_{3}$ and $\hat{s}_{3}$ in the final state. It is easy to verify that this correspondence can be achieved under the action of the Hamiltonian

$$
\hat{H}=\hat{\sigma}_{1} \frac{\hat{s}_{1} \cos \phi+\hat{s}_{3} \sin \phi}{\sqrt{2}}+\frac{\left(\hat{s}_{2}-\hat{s}_{1}\right) \sin \phi+\hat{s}_{3} \cos \phi}{2},
$$

where $2 \phi=0.95531$ is the angle between $\vec{v}_{++}$and the $z$-axis, that is, $\cos 2 \phi=1 / \sqrt{3}$. Noting that $\hat{H}^{2}=\sin ^{2} \chi$, where $\chi=1.11069$ satisfies $\cos \chi=\frac{1}{2} \cos \phi$, we obtain $\hat{U}=\mathrm{e}^{-\mathrm{i} \hat{H} \tau}=\cos (\tau \sin \chi)-i \hat{H} \sin (\tau \sin \chi) / \sin \chi$. Taking as duration of the evolution $\tau=\chi / \sin \chi$, we obtain $\hat{U}=$ $\cos \chi-i \hat{H}$. Insertion in (3) allows to check Eq. (6) and to get the expected optimal correspondence (77).

The simpler Hamiltonian

$$
\hat{H}=\frac{1}{\sqrt{2}} \hat{\sigma}_{1} \hat{s}_{1}+\frac{1}{2}\left(\hat{s}_{2} \sin \phi+\hat{s}_{3}\right),
$$

which results from (8) through rotation of $\hat{\vec{s}}$, also achieves an optimal mapping $\hat{\rho} \mapsto P_{\alpha}$, provided $\hat{s}_{3}$ is replaced by $\hat{s}_{1} \sin \phi+\hat{s}_{3} \cos \phi$ both in the measured projections $\hat{p}_{a}=\frac{1}{2}\left(1 \pm \hat{s}_{3}\right)$ and in the initial state $\hat{r}=\hat{p}_{+}$. The first term of Eq. (9) describes, in the spin language, an Ising coupling, while the second term represents a transverse magnetic field acting on A only.

Larger assistant. We have optimized above the determination of $\hat{\rho}$ by coupling S to an assistant A that starts in a pure state and has the same dimension $n=m=2$ as S. It is natural to wonder whether the quality of the measurement, as expressed by the magnitude of $\Delta$, may be improved for $n>m=2$ and/or for mixed initial states of A. We may, for instance, consider an assistant consisting of $q$ spins, in which case $m=2$ and $n=2^{q}$. We now denote as $\hat{s}_{3}$ some two-valued observable of A which is subjected to measurement at the time $\tau$. The only changes in (11) are the dimension $n$ of the matrix $\hat{r}$ and the fact that the two projection operators $\hat{p}_{a}$ no longer constitute a complete set in the Hilbert space of A. Experiment still provides the four probabilities $P_{\alpha}=\operatorname{tr} \hat{\mathcal{R}}_{\tau}\left(\hat{\pi}_{i} \otimes \hat{p}_{a}\right)$ with unit sum, where $\hat{\mathcal{R}}_{\tau}=\hat{U}(\hat{\rho} \otimes \hat{r}) \hat{U}^{\dagger}$ is the final density operator in the $m n$-dimensional space of $\mathrm{S}+\mathrm{A}$, and the mapping $\hat{\rho} \mapsto P_{\alpha}$ keeps the form (2). The conditions (4) still hold, since they express simply that the correspondence (11) or (2) preserves the positivity and the normalization. When obtaining the upper bound $1 /(12 \sqrt{3})$ for $|\Delta|$ we relied only on these conditions. Therefore, using a larger assistant cannot improve upon the optimal solutions found for $n=2$ and pure $\hat{r}$.
In all cases, the maximum of $|\Delta|$ is reached for mappings (2 13) which involve the regular tetrahedron (6). In such mappings there exist 4 pure states, $\hat{\rho}_{\bar{\alpha}}=\frac{1}{2}\left(1-4 \vec{v}_{\bar{\alpha}}\right.$. $\hat{\vec{\sigma}}$ ), for which one probability, $P_{\bar{\alpha}}$, vanishes.

Completely disordered assistant. Returning to the case $n=m=2$ we saw that we could reach the upper bound $1 /(12 \sqrt{3})$ of $|\Delta|$ if the assistant is initially in a pure state. In order to explore how much is lost if it is in a mixed state, we consider the extreme situation in which $\hat{r}=\frac{1}{2} \hat{1}$ is the completely disordered state. There is an advantage in using such a state, as it is easier to prepare than a pure state: one lets the assistant interact with a hot thermal bath; for a spin, one leaves it unpolarized. Eqs. (22 3) taken for $\hat{r}=\frac{1}{2} \hat{1}$ show that too simple evolutions may lead to a vanishing determinant $\Delta$. For instance, the above evolution (8), which we optimized for $\hat{r}=\hat{p}_{+}$, is completely ineffective for $\hat{r}=\frac{1}{2} \hat{1}$, since it maps any $\hat{\rho}$ onto the trivial set of probabilities $P_{\alpha}=\frac{1}{4}$.

In order to maximize $|\Delta|$ with respect to $\hat{U}$ for $\hat{r}=\frac{1}{2} \hat{1}$, we now have to evaluate the vectors $\vec{v}_{\alpha}$ from (3). We only sketch this calculation here. We represent $U_{a i, b j}$ as $U_{+\cdot,+}=V K, U_{-\cdot,+}=W K^{\prime}, U_{+\cdot,-\cdot}=V K^{\prime} X$, $U_{-\cdot,-}=-W K X$, in terms of two hermitian positive $2 \times 2$ matrices $K$ and $K^{\prime}$ such that $K^{2}+K^{\prime 2}=1$, and of three unitary $2 \times 2$ matrices $V, W, X$ in the space $i= \pm, j= \pm$ of S. Using the invariances of the problem, we may without restrictions parametrize $K=K(\theta, \varphi) \equiv$ $\cos \theta \cos \varphi+\sin \theta \sin \varphi \vec{\chi} \cdot \overrightarrow{\hat{\sigma}}$ with unit vector $\vec{\chi}$, implying $K^{\prime}=K\left(\theta-\frac{\pi}{2}, \varphi\right)$ and $K^{2}-K^{\prime 2}=K(2 \theta, 2 \varphi)$. We characterize $V$ by $V^{\dagger} \hat{\sigma}_{3} V=\vec{\eta} \cdot \overrightarrow{\hat{\sigma}}, W$ by $W^{\dagger} \hat{\sigma}_{3} W=\vec{\zeta} \cdot \overrightarrow{\hat{\sigma}}$, and take $X=\vec{\xi} \cdot \overrightarrow{\hat{\sigma}}$, where also $\vec{\eta}, \vec{\zeta}, \vec{\xi}$ are unit vectors, with $\vec{\chi} \cdot \vec{\xi}=0$. We then obtain $u_{\alpha}=\frac{1}{4}$ and the $\vec{v}_{\alpha}$, whence

$$
\Delta=\frac{1}{32} \sin 4 \theta \sin 4 \varphi(\vec{\chi} \times \vec{\xi}) \cdot[(\vec{\zeta} \cdot \vec{\xi}) \vec{\eta}+(\vec{\eta} \cdot \vec{\xi}) \vec{\zeta}] .
$$

We now determine the maximum value of $|\Delta|$ attainable for $\hat{r}=\frac{1}{2} \hat{1}$ and the corresponding optimal evolution $\hat{U}$. The best angles are $\theta=\varphi=\pi / 8$. Choosing $\vec{\chi}=(1,0,0)$ and $\vec{\xi}=(0,1,0)$, we see that the largest $|\Delta|$ is $1 / 32$. This still corresponds to $\vec{v}_{\alpha}$ forming a regular tetrahedron, but with $\left|\vec{v}_{\alpha}\right|=\sqrt{3} / 8$ instead of $\left|\vec{v}_{\alpha}\right|=\frac{1}{4}$. Accordingly, for the same orientation of the $\vec{v}_{\alpha}$ 's, the factor $\sqrt{3}$ in (17) is replaced by 2 . Rather surprisingly, the efficiency of the new scheme is not much worse than when the assistant is prepared in a pure state. However, the Hamiltonians needed now to maximize $\Delta$ require a more complicated coupling than in (8). Among various possibilities, we present here an example:

$$
\hat{H}=\frac{1}{2} \overrightarrow{\hat{\sigma}} \cdot \overrightarrow{\hat{s}}-\sqrt{2} \hat{\sigma}_{2} \hat{s}_{2}+\frac{1}{\sqrt{2}}\left(\hat{\sigma}_{1}+\hat{s}_{1}\right)
$$

(the signs of the three terms can be changed independently). This Hamiltonian involves an anisotropic Heisenberg interaction and an external field acting symmetrically on S and A. Using Eq. (3) with $\hat{r}=\frac{1}{2} \hat{1}$, one can check that for $\tau=\frac{1}{4}(2 k+1) \pi$ the evolution operator 
$\hat{U}=\mathrm{e}^{-i \hat{H} \tau}$ leads to an optimal solution with $\Delta=1 / 32$. For $\tau=\pi / 4$ Eq. (7) is replaced by

$$
\begin{gathered}
\rho_{1}=2\left\langle\hat{\sigma}_{3} \hat{s}_{3}\right\rangle, \quad \rho_{2}=2\left(\left\langle\hat{s}_{3}\right\rangle \cos \gamma+\left\langle\hat{\sigma}_{3}\right\rangle \sin \gamma\right), \\
\rho_{3}=2\left(\left\langle\hat{\sigma}_{3}\right\rangle \cos \gamma-\left\langle\hat{s}_{3}\right\rangle \sin \gamma\right), \quad \gamma=\frac{\pi(1+\sqrt{2})}{4} .
\end{gathered}
$$

Conclusion. The non-commutative information contained in the density matrix of quantum system can be transformed by a one-to-one correspondence into ordinary information associated with a set $P_{\alpha}$ of ordinary probabilities for exclusive events. The price to be paid is the introduction of our extra assistant system A. This correspondence can be experimentally implemented by repeatedly letting $\mathrm{S}$ and A suitably interact, then by performing each time simultaneous measurements of two commuting observables $\hat{\omega}$ and $\hat{o}$ pertaining to $\mathrm{S}$ and $\mathrm{A}$, respectively. Counting of events yields the probabilities $P_{i a}$ for $\hat{\omega}$ to take the value $\omega_{i}$ and for $\hat{o}$ to take the value $o_{a}$. Provided $\hat{\omega}$ is non degenerate so that its number of distinct eigenvalues $\omega_{i}(1 \leq i \leq m)$ equals the dimension $m$ of the Hilbert space of $\mathrm{S}$, and provided the number of eigenvalues of $\hat{o}$ is also $m$ (at least), the correspondence $\hat{\rho} \mapsto P_{\alpha}$ can be inverted, as we displayed on several examples. The second condition implies that the assistant has a dimension $n \geq m$. Hence an initially unknown $\hat{\rho}$ can be determined via $P_{\alpha}$ by means of a single apparatus.

As compared to the standard determination schemes of $\hat{\rho}$ based on direct non-commutative measurements of $\mathrm{S}$, the present method has several advantages. i) It is more economical, since it involves only one observable $\hat{\omega}$ of $\mathrm{S}$ and one observable $\hat{o}$ of $\mathrm{A}$, whereas direct determinations require measuring at least $m+1$ non-commuting observables of S. ii) This full set of $m+1$ observables is not always accessible in practice. For instance, for a two-level atom prepared in some unknown state $\hat{\rho}, \rho_{3}$ is readily measured through the occupation probability of the excited state, but $\rho_{1}$ and $\rho_{2}$ can be determined only indirectly. Interaction of $\mathrm{S}$ with another initially known two-level atom A (whose own preparation may be straightforward, as we saw above) may provide the full $\hat{\rho}$ through mere simultaneous measurements of the occupation probabilities for the levels of $\mathrm{S}$ and $\mathrm{A}$. iii) It has been stressed $^{10}$ that the use of standard statistical and information theoretical methods for dealing with incomplete or noisy experimental data cannot be directly extended to quantum mechanics, because results are produced there by means of non-commutative measurements; indeed, these data pertain to different contexts as they are produced by different apparatuses. The present scheme, involving only commutative measurements, circumvents this difficulty.

Taking as a criterion of quality of our measurement schemes the size of the determinant of the mapping $\hat{\rho} \mapsto P_{\alpha}$, we have explored for $m=2$ the conditions that lead to the best determination of $\hat{\rho}$ for some uncertainty on the set $P_{\alpha}$. For an assistant with dimension 2 , its known initial state $\hat{r}$ should be pure and the pa- rameters of the Hamiltonian should be suitably chosen. An example of an optimal evolution is generated by the Hamiltonian (8) or (9). We have also seen that the determinant cannot be enlarged by use of an assistant with dimension larger than 2. (However, $\hat{o}$ may then take more than two eigenvalues $o_{a}$ and the probabilities $P_{i a}$ become more numerous than needed; the fact that they are related to one another independently of $\hat{\rho}$ opens the possibility of improving the determination of $\hat{\rho}$ through cross-check of the data $P_{\alpha}$.)

For $m=2$, the optimal mappings (2), (6) amount to identify, via a dynamical process, the joint probabilities $P_{\alpha}$ for $\hat{\sigma}_{3}$ and $\hat{s}_{3}$ with the expectation values in the state $\rho$ of the observables $\frac{1}{2}\left(\hat{1}-\hat{\Omega}_{\alpha}\right)$, where $\hat{\Omega}_{\alpha}=\frac{1}{2}\left(\hat{1}-4 \vec{v}_{\alpha} \cdot \overrightarrow{\hat{\sigma}}\right)$ pertains to the system S. The 4 observables $\hat{\Omega}_{\alpha}$ are projection operators, satisfying $\operatorname{tr} \hat{\Omega}_{\alpha} \hat{\Omega}_{\beta}=\frac{1}{3}$ for $\alpha \neq \beta$, and spanning the space of observables $\hat{\omega}$ of S. For $m>2$ we conjecture that a bound on $\Delta$ may be found by considering in the Hilbert space of $\mathrm{S}$ a set of $\mathrm{m}^{2}$ projections $\hat{\Omega}_{\alpha}$, satisfying $\operatorname{tr} \hat{\Omega}_{\alpha}=1, \operatorname{tr} \hat{\Omega}_{\alpha} \hat{\Omega}_{\beta}=1 /(m+1)$ for $\alpha \neq \beta, \sum_{\alpha} \hat{\Omega}_{\alpha}=m \hat{1}$, and constituting a basis for the observables $\hat{\omega}$. Then the mapping matrix $R$ in $P_{\alpha}=\sum_{i j} R_{\alpha, i j} \rho_{j i}$ is expected to be given by $m(m-1) R_{\alpha, i j}=\delta_{i j}-\Omega_{\alpha, i j}$. This form makes the determinant $\Delta$ stationary under the constraints imposed by positivity and normalization alone. As above, there are $m^{2}$ pure states $\hat{\rho}_{\bar{\alpha}}=\hat{\Omega}_{\bar{\alpha}}$ for which one probability, $P_{\bar{\alpha}}$, vanishes. This conjecture yields for $\Delta^{2}$ the upper bound $m^{-1}\left[m(m+1)(m-1)^{2}\right]^{1-m^{2}}$, which generalizes the $m=2$-result $\Delta^{2}=1 /(12 \sqrt{3})^{2}$.

It might have been expected that a completely disordered assistant makes the determination of $\hat{\rho}$ from observation of $P_{\alpha}$ unprecise or even infeasible; for $m=2$ it turns out that the best Jacobian in this case is smaller than the maximum one only by a factor $(\sqrt{3} / 2)^{3} \simeq 0.65$, and that Hamiltonians as simple as (11) can be used.

Indeed, the various types of two-level systems on which experiments are currently performed (NMR, quantum and atomic optics, spintronics) feature Hamiltonians similar to (9) and (11) that optimize the process, with Ising or Heisenberg types of couplings. For instance, the spinspin interaction between two single-electron quantum dots is usually anisotropic due to spin-orbit coupling or to a lack of symmetry of the host material; see 11 for a recent discussion. Experiments can therefore easily be designed along the above ideas. They will demonstrate that the principle of complementarity, which seems to imply that different measurement devices are needed to fully determine a quantum state, can be by-passed by using an assistant, even completely disordered.

The work of A.E. A is part of the research programme of the Stichting voor Fundamenteel Onderzoek der Materie (FOM, financially supported by the Nederlandse Organisatie voor Wetenschappelijk Onderzoek (NWO)). R. B. acknowledges hospitality at the University of Amsterdam, and A.E. A. and Th.M. N. at the CEA-Saclay. 
1 E.C. Kemble, Fundamental Principles of Quantum Mechanics, (McGraw-Hill, New York, 1937).

2 W. Pauli, Encyclopedia of Physics, (Springer-Verlag, Berlin, 1958), volume V, p. 17.

3 U. Leonhardt, Measuring the Quantum State of Light, (Cambridge University Press, 1997).

4 R. G. Newton and B.-L. Young, Ann. Phys. 49, 393 (1968). W. Band and J.L. Park, Am. J. Phys., 47, 188 (1979). S. Weigert, Phys. Rev. A 45, 7688 (1992). I.D. Ivanovic, J. Phys. A 26, L579 (1993). O. Steuernagel and J.A. Vaccaro, Phys. Rev. Lett. 75, 3201 (1995). G. Klose, et. al., Phys. Rev. Lett. 86, 4721 (2001). R. Asplund and G. Björk, Phys. Rev. A 64, 012106 (2001).
5 N. Bohr, Phys. Rev., 48, 696 (1935).

${ }^{6}$ C.W. Helstrom, Quantum Detection and Estimation Theory, (Academic Press, New York, 1976).

7 H.P. Yuen, quant-ph/0109054 A. Chefles, quant-ph/0010114 R. Gill and M. Guta, quant-ph/0303020

8 G.M. D'Ariano, Phys. Lett. A 300, 1 (2002)

9 A.E. Allahverdyan, R. Balian and Th.M. Nieuwenhuizen, unpublished.

10 C. Brukner and A. Zeilinger, Phys. Rev. A, 63, 022113 (2001). P.G.L. Mana, quant-ph/0302049

11 K.V. Kavokin, Phys. Rev. B, 64, 075305 (2001). 